This is the author's copy of the publication as archived with the DLR's electronic library at http://elib.dlr.de . Please consult the original publication for citation.

\title{
Nonlinear Control of Dual Half Bridge Converters in Hybrid Energy Storage Systems
}

\author{
Ricardo de Castro, Jonathan Brembeck, Rui Esteves Araujo
}

This work proposes a new control framework for power converters with a dual half bridge (DHB) configuration. The new framework exploits the multi-port structure of the DHB to simultaneously: i) regulate the current in the primary side of the DHB and ii) equalize the voltage in the two secondary ports of the DHB. To implement these functions, we combine input-output linearization methods with pragmatic voltage balance algorithms. We then apply this framework to a hybrid energy storage system composed of a battery pack and two supercapacitor modules. Numerical simulation results demonstrate the effectiveness of the proposed approach in regulating the power between the energy storage units and balancing the supercapacitors' voltages.

\section{Copyright Notice}

(C2020 IEEE. Personal use of this material is permitted. Permission from IEEE must be obtained for all other uses, in any current or future media, including reprinting/republishing this material for advertising or promotional purposes, creating new collective works, for resale or redistribution to servers or lists, or reuse of any copyrighted component of this work in other works.

R. de Castro, J. Brembeck, R.E. Araujo: Nonlinear Control of Dual Half Bridge Converters in Hybrid Energy Storage Systems, 2020 IEEE Vehicle Power and Propulsion Conference, VPPC 2020 - Proceedings 


\title{
Nonlinear Control of Dual Half Bridge Converters in Hybrid Energy Storage Systems
}

\author{
Ricardo de Castro*, Jonathan Brembeck*, Rui Esteves Araujo ${ }^{\dagger}$, \\ * Institute of System Dynamics and Control, German Aerospace Center (DLR), Wessling, D-82234, Germany \\ ${ }^{\dagger}$ INESC TEC and Faculty of Engineering, University of Porto, Porto, 4200-465, Portugal
}

\begin{abstract}
This work proposes a new control framework for power converters with a dual half bridge (DHB) configuration. The new framework exploits the multi-port structure of the DHB to simultaneously: i) regulate the current in the primary side of the DHB and ii) equalize the voltage in the two secondary ports of the DHB. To implement these functions, we combine input-output linearization methods with pragmatic voltage balance algorithms. We then apply this framework to a hybrid energy storage system composed of a battery pack and two supercapacitor modules. Numerical simulation results demonstrate the effectiveness of the proposed approach in regulating the power between the energy storage units and balancing the supercapacitors' voltages.

Index Terms-power conversion, nonlinear control, dual half bridge converters, hybrid energy storage
\end{abstract}

\section{INTRODUCTION}

Energy storage systems (ESSs) are usually composed of heterogeneous storage elements. This heterogeneity might result from an intentional design decision to integrate diverse types of storage elements, such as batteries, supercapacitors and/or fuel cells [1]. It can also result from aging mechanisms, such as cell-to-cell variations that occur during the lifetime of a large battery pack [2]. To effectively deal with this heterogeneity, ESSs need to incorporate power conversion functions. These functions are key to actively balancing large battery packs or performing energy management in hybrid ESSs [1].

Dual half bridge (DHB) configurations are an attractive solution to implement power conversion functions. In addition to galvanic isolation, zero-voltage switching, and bidirectional power flow [3], they also present a reduced number of switches when compared to dual full bridge configurations [4]. Because of these attractive features, DHB has been employed in multiple applications, including active battery balancing [5], battery chargers [6] and DC micro grids [7].

This work provides two main contributions. The first is related to the development of a nonlinear control framework for DHB converters, based on input-output linearization. This approach cancels the dominant nonlinearities in the converter, providing a strong contrast with previous works on DHB control, which rely almost exclusively on linear methods [7][10] with limited validity range. In comparison with these linear approaches, the nonlinear framework proposed here improves control performance when the DHB is subject to large signal variations. It also offers an effective mechanism to compensate for couplings in the control inputs of the converter.

The second contribution exploits the multiple degrees of freedom offered by the DHB to improve power conversion in hybrid ESSs. To better understand this contribution, it is worth mentioning that the original DHB configuration proposed by [3], [8] was initially treated as a 2-port converter with one controllable degree of freedom, the phase shift. More recently, this configuration has been extended in new directions. For example, [10] and [11] exploited a second degree of freedom (the converter's duty cycle) to reduce the transformer's current and energy losses, while [4] studied the steady-state operation of the DHB as a 3- and 4-port power converter.

Inspired by these recent works, we investigate here the possibility of using the DHB in a 3-port configuration to regulate the power flow in hybrid ESSs composed of batteries and supercapacitors (SCs). Our idea consists in connecting the battery module to the primary side of the DHB, while the (two) secondary ports of the DHB are connected to two SC modules. This 3-port arrangement allows us to simultaneously perform two functions: i) exchange power from the primary (battery) and secondary (SC) sides through manipulation of the phase shift; and ii) balance voltage/capacity imbalances that might exist in the two SCs modules via manipulation of the converter's duty cycle. The first function is useful to implement energy-management strategies for the hybrid ESSs, e.g. for reducing battery stress and aging [1], [5]; the second prevents over-voltage conditions in the SCs with heterogeneous properties or initial conditions, and guarantees that the SC energy is fully used [12]. While a conventional DHB with 2-port configuration could also be employed for converting power within the hybrid ESSs, the 3-port variant proposed here allows us to double the voltage of the SC pack without requiring additional balancing hardware.

\section{Converter Model}

\section{A. Equivalent-circuit Model}

Fig. 1 shows the equivalent circuit of the DHB. It relies on the configuration proposed by [8] with one key modification: SCs are employed in the secondary side $\left(C_{s c 1}, C_{s c 2}\right)$ instead of regular capacitors. This allows a greater integration between the converter and the hybrid ESS, decreasing the number of components. Additionally, to capture self discharge of the SCs, two current sources $\left(i_{s c 1}, i_{s c 2}\right)$ are included in parallel with the SCs.

The converter's primary side is connected with the battery $\left(v_{b}\right)$, an input inductor $L_{b}$ with parasitic resistance $R_{b}$ and two capacitors $C_{1}, C_{2}$. A transformer links the primary and secondary sides of the converter. To facilitate the physical and 


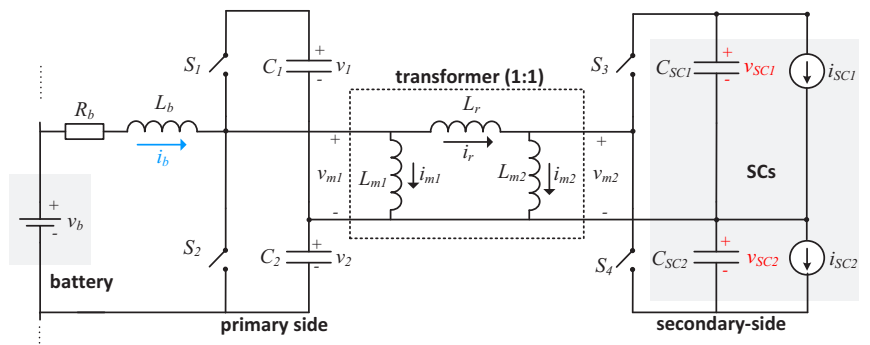

Fig. 1. Equivalent circuit of the dual half-bridge converter and its integration with the energy storage units (batteries and SCs).

mathematical analysis of the DHB, this transformer is modeled here in a $\pi$ representation [13], with one leakage inductance $\left(L_{r}\right)$ and two shunt magnetization branches $\left(L_{m 1}, L_{m 2}\right)$,

The converter is controlled via the (on/off) position of the primary $\left(S_{1}, S_{2}\right)$ and secondary $\left(S_{3}\right.$ and $\left.S_{4}\right)$ switches. They operate with a switching frequency $\omega$ and duty cycle $d$. A phase shift of $\phi[\mathrm{rad}]$ delays the modulation signals of the primary and secondary sides (see Fig. 2), enabling the power flow between the battery and the SCs. Since $S_{2}$ and $S_{4}$ have complementary operation w.r.t. $S_{1}$ and $S_{3}$, the DHB has four operating modes $(j \in\{1,2,3,4\})$, which depend on the positions of the switches (see Fig. 2). For each mode, the dynamics of the capacitors' voltages $\left(v_{1}, v_{2}, v_{s c 1}, v_{s c 2}\right)$ and inductors' currents $\left(i_{b}, i_{r}, i_{m 1}, i_{m 2}\right)$ is given by:

$$
\begin{aligned}
\frac{d x}{d t} & =A_{s x}^{j} x+A_{s r}^{j} i_{r}+A_{s m}^{j} i_{m}+B_{s}^{j} v_{b}+E_{s}^{j} i_{s c} \\
\frac{d i_{r}}{d t} & =A_{f x}^{j} x \\
\frac{d i_{m}}{d t} & =A_{m x}^{j} x
\end{aligned}
$$

where $x=\left[\begin{array}{lllll}i_{b} & v_{1} & v_{2} & v_{s c 1} & v_{s c 2}\end{array}\right]^{T}, i_{r}$ is the current in the leakage inductance and $i_{m}=\left[\begin{array}{ll}i_{m 1} & i_{m 2}\end{array}\right]^{T}$ the magnetization current vector. The battery voltage $v_{b}$ and the SC self-discharge currents $i_{s c}=\left[\begin{array}{ll}i_{s c 1} & i_{s c 2}\end{array}\right]^{T}$ represent exogenous inputs to the system. The constant matrices $\left(A_{s x}^{j}, A_{s r}^{j}, A_{s m}^{j}, B_{s}^{j}, E_{s}^{j}, A_{f x}^{j}, A_{m x}^{j}\right)$ can be obtained via circuit analysis for each mode $j$ and are omitted here for the sake of brevity.

\section{B. Averaged Model}

To facilitate the construction of the averaged model of the DHB, we follow the approach proposed by [14] and analytically compute the solution for the leakage current $i_{r}$. Assuming $x$ varies slowly over one switching period, a closedform solution $i_{r}^{*}(t, x)$ can be obtained ${ }^{1}$ and incorporated into (1a)

$$
\frac{d x}{d t}=A_{s x}^{j} x+A_{s r}^{j} i_{r}^{*}(t, x)+A_{s m}^{j} i_{m}+B_{s}^{j} v_{b}+E_{s}^{j} i_{s c}
$$

\footnotetext{
${ }^{1}$ omitted here due to space constraints
}

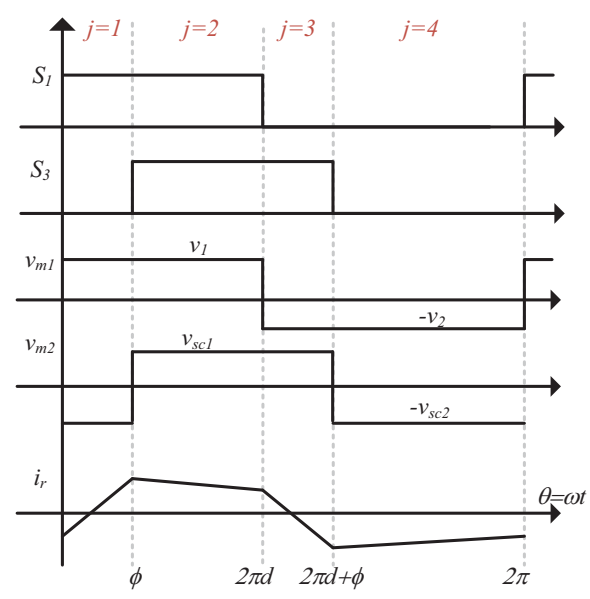

Fig. 2. Illustration of the four operating modes of the DHB converter and their effect in the voltage and current of the leakage inductor.

The dynamics of the average value of $x$ and $i_{m}$ (denoted $X$ and $I_{m}$ ) can be obtained by averaging the right hand side of (1a), (1c) over one switching period [14]:

$$
\begin{aligned}
\frac{d X}{d t} & =A_{s x}(u) X+B_{s}(u) V_{b}+A_{s m}(u) I_{m}+E_{s}(u) I_{s c} \\
\frac{d I_{m}}{d t} & =A_{m x}(u) X=\left[\begin{array}{ccccc}
0 & \frac{d}{L_{m 1}} & \frac{1-d}{L_{m 1}} & 0 & 0 \\
0 & 0 & 0 & \frac{d}{L_{m 2}} & \frac{1-d}{L_{m 2}}
\end{array}\right] X
\end{aligned}
$$

where $u=\left[\begin{array}{ll}d & \phi\end{array}\right]^{T}$ is the control input (dependent on the phase shift $\phi$ and duty cycle $d$ ), $X=\left[\begin{array}{lllll}I_{b} & V_{1} & V_{2} & V_{s c 1} & V_{s c 2}\end{array}\right]^{T}$ the average states, and $A_{s x}(u), B_{s}(u), A_{s m}(u), E_{s}(u), A_{m x}(u)$ matrices with polynomial dependence on $u$, omitted here for the sake of brevity. A word on the notation: in this work, average values of states and variables are represented in upper case.

\section{Reduced-order Model}

To derive a practical model for the DHB, a couple of practical assumptions will be adopted. First, we assume that the average magnetization currents $I_{m}$ operate close to quasistatic conditions, i.e. $d I_{m} / d t \approx 0$. We also assume that both the magnetization and self-discharge currents of the SCs are relatively small and can be neglected for control purposes, thus $I_{s c} \approx 0, I_{m} \approx 0$. These approximations can be justified by the large impedance of the (shunt) magnetization branches of the transformer and the high self-discharge resistance of the SCs. Introducing these approximations into the average model yields a differential-algebraic system of equations:

$$
\begin{aligned}
\frac{d X}{d t} & \approx A_{s x}(u) X+B_{s}(u) V_{b} \\
0 & \approx A_{m x}(u) X
\end{aligned}
$$

To further simplify the model, we focus on the dynamic evolution of the summation of voltages in the primary capacitors $\left(V_{12}\right)$ and in the SCs $\left(V_{s c}\right)$ :

$$
V_{1}+V_{2}=V_{12}, \quad V_{s c 1}+V_{s c 2}=V_{s c}
$$




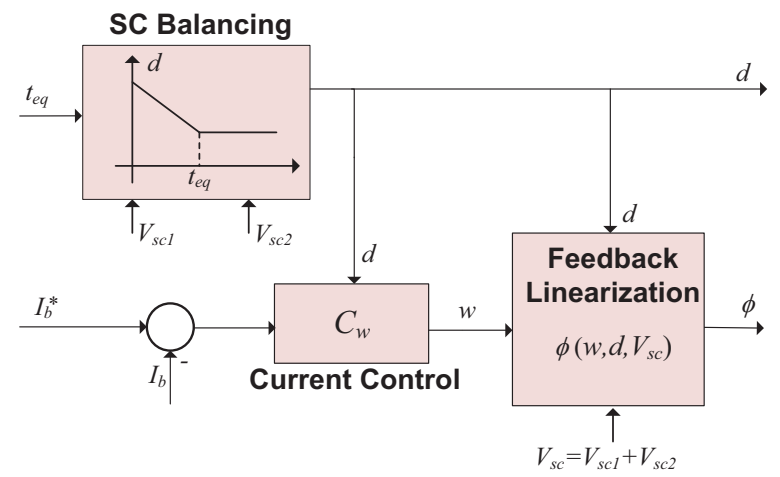

Fig. 3. Block diagram of the control algorithm.

Combining these equations with (5) and assuming symmetric capacitances in the primary $\left(C_{b}=C_{1}=C_{2}\right)$ and secondary $\left(C_{s c}=C_{s c 1}=C_{s c 2}\right)$ sides, allows us to obtain the following reduced-order model:

$$
\begin{aligned}
\frac{d I_{b}}{d t} & =-\frac{d}{L_{b}} V_{12}+\frac{1}{L_{b}} V_{b} \\
\frac{d V_{12}}{d t} & =\frac{2 d}{C_{b}} I_{b}+\frac{\phi(4 \pi d(d-1)+\phi)}{2 C_{b} L_{r} \omega \pi} V_{s c} \\
\frac{d V_{s c}}{d t} & =-\frac{\phi(4 \pi d(d-1)+\phi)}{2 C_{s c} L_{r} \omega \pi} V_{12} \\
y & =\left[\begin{array}{c}
I_{b} \\
V_{1} \\
V_{2} \\
V_{s c 1} \\
V_{s c 2}
\end{array}\right]=\left[\begin{array}{ccc}
1 & 0 & 0 \\
0 & (1-d) & 0 \\
0 & d & 0 \\
0 & 0 & (1-d) \\
0 & 0 & d
\end{array}\right]\left[\begin{array}{c}
I_{b} \\
V_{12} \\
V_{s c}
\end{array}\right]
\end{aligned}
$$

This reduced model contains 3 states, 5 outputs, 2 control inputs $(d, \phi)$ and 1 exogenous input $\left(V_{b}\right)$, providing a compact, control-oriented representation of the DHB.

\section{Controller Design}

The DHB control is divided into two loops: i) balancing of $\mathrm{SC}$ voltage and ii) control of the converter's input current.

\section{A. Balancing of the SC Voltage}

The goal of the SC balancing task is to reach equalization of the SCs voltages in less than $t_{e q}$, the desired equalization time. This means:

$$
\frac{V_{s c 1}(t)}{V_{s c 2}(t)}=1, \quad t \geq t_{e q}
$$

Inspecting the output model (7d), one can find the duty cycle $d$ has a direct impact in $V_{s c 1}, V_{s c 2}$, particularly their ratio:

$$
\frac{V_{s c 1}(t)}{V_{s c 2}(t)}=\frac{1-d(t)}{d(t)}
$$

This relation motivates us to employ $d$ as a manipulated variable in the balancing task.

Let us now discuss a practical algorithm for generating the duty cycle. When we start the system at $t=t_{0}$ the SCs might have different voltages, e.g. due to variations in self-discharge currents. To prevent large current transient at the start, it is convenient to match the initial duty cycle with the initial SCs voltages:

$$
\frac{V_{s c 1}\left(t_{0}\right)}{V_{s c 2}\left(t_{0}\right)}=\frac{1-d\left(t_{0}\right)}{d\left(t_{0}\right)} \Rightarrow d\left(t_{0}\right)=\left(1+\frac{V_{s c 1}\left(t_{0}\right)}{V_{s c 2}\left(t_{0}\right)}\right)^{-1}
$$

On the other hand, to enforce the desired equalization time, we need to reach voltage equalization of the SCs in $t_{e q}$, hence

$$
\frac{V_{s c 1}(t)}{V_{s c 2}(t)}=1=\frac{1-d(t)}{d(t)} \Rightarrow d(t)=\frac{1}{2}, \forall t \geq t_{e q}
$$

This equation together with (10) define two boundary conditions for the duty cycle, $d\left(t_{0}\right)$ and $d\left(t_{e q}\right)$. We propose a linear relation to connect these two boundary conditions, which leads to the following algorithm for SC balancing:

$$
d(t)= \begin{cases}\left(1+\frac{V_{s c 1}\left(t_{0}\right)}{V_{s c 2}\left(t_{0}\right)}\right)^{-1} & t=t_{0} \\ \left(1+\frac{V_{s c 1}\left(t_{0}\right)}{V_{s c 2}\left(t_{0}\right)}\right)^{-1}+\frac{\frac{1}{2}-d\left(t_{0}\right)}{t_{e q}-t_{0}}\left(t-t_{0}\right) & t_{0}<t<t_{e q} \\ 1 / 2 & t \geq t_{e q}\end{cases}
$$

This algorithm can be seen as a "soft start" strategy to slowly balance the SC voltage. It avoids surge power transients in the converter during the initialization phase, as long as $t_{e q}$ is sufficiently large.

\section{B. Current Control}

1) Virtual Input: The design of the current controller relies on feedback linearization [15]. It seeks to cancel the nonlinearities between the control inputs $(d, \phi)$ and the output $I_{b}$. Inspecting (7), one can find that $\phi(4 \pi d(d-1)+\phi)$ represents the main nonlinear term in the state-space model. This motivate us to define the following change of variable

$$
w=\phi(4 \pi d(d-1)+\phi) V_{s c}
$$

where $w$ is a virtual control input. Introducing $w$ in (7) yields:

$$
\begin{aligned}
\frac{d I_{b}}{d t} & =-\frac{d}{L_{b}} V_{12}+\frac{1}{L_{b}} V_{b} \\
\frac{d V_{12}}{d t} & =\frac{2 d}{C_{b}} I_{b}+\frac{1}{2 C_{b} L_{r} \omega \pi} w \\
\frac{d V_{s c}}{d t} & =-\frac{1}{2 C_{s c} L_{r} \omega \pi} \frac{V_{12}}{V_{s c}} w \\
y_{1} & =I_{b}
\end{aligned}
$$

In this representation, the primary-side states $\left(I_{b}, V_{12}\right)$ have linear dynamics (under the standing assumption of a slowvarying $d$ ), while the secondary-side state $\left(V_{s c}\right)$ has a nonlinear behavior. This allows us to obtain the following transfer functions that relate $\left(w, V_{b}\right)$ with the current $I_{b}$ :

$$
\begin{aligned}
I_{b}(s) & =\frac{\alpha_{w}(d)}{1+\frac{s^{2}}{\omega_{n}^{2}(d)}} w(s)+\frac{\alpha_{v}(d) s}{1+\frac{s^{2}}{\omega_{n}^{2}(d)}} V_{b}(s) \\
& =G_{w}(s, d) w(s)+\underbrace{G_{v}(s, d) V_{b}(s)}_{\sigma(s)}
\end{aligned}
$$


TABLE I

SPECIFICATIONS OF THE CURRENT CONTROLLER.

\begin{tabular}{|c|c|c|}
\hline Parameter & Symbol & Value \\
\hline max. real pole & $\bar{s}_{r}$ & $-2 \pi 20 \mathrm{rad} / \mathrm{s}$ \\
low-frequency model uncertainty & $l_{T}$ & 0.5 \\
high-frequency model uncertainty & $h_{T}$ & 1.5 \\
cross-over frequency for model uncertainty & $\omega_{T}$ & $1.6 \omega_{n}(d)$ \\
\hline
\end{tabular}

where $\omega_{n}^{2}(d)=2 d^{2} /\left(L_{b} C_{b}\right)$ is the natural frequency and $\alpha_{w}(d)=\left(4 L_{r} \omega \pi d\right)^{-1}, \alpha_{v}(d)=C_{b} /\left(2 d^{2}\right)$ gains. To facilitate the controller design, $V_{b}$ is treated as a generic output disturbance $(\sigma(s))$ that the controller needs to reject.

2) Controller Structure: As depicted in Fig. 3, the current controller is divided into two parts. The first relies on a linear controller that manipulates the virtual control input $w$ as a means to track the reference current $I_{b}^{*}$. The second computes the desired phase shift $\phi$ through inversion of $w$. The linear controller, called w-controller in the sequel, is composed of an integrator and two zeros:

$w(s, d)=C_{w}(s, d) e(s)=\frac{1}{\alpha_{w}(d)} k_{c} \frac{s^{2}+2 \zeta_{z} \omega_{z} s+\omega_{z}^{2}}{s} e(s)$

where $e(s)=I_{b}^{*}(s)-I_{b}(s)$ is the tracking error, $C_{w}$ the transfer function of the controller, $k_{c}$ the controller's gain, $\omega_{z}$ the natural frequency of the zeros and $\zeta_{z}$ their damping.

After computing the virtual control input $w$, the desired phase shift can be obtained via inversion of the quadratic mapping (12).

3) Specifications: The $w$-controller is subjected to transient response and robustness specifications.

The transient response specification limits the region where the closed-loop poles of the current response can lie. In particular, we specify that the real part of the poles of $I_{b}(s)=T(s, d) I_{b}^{*}$ should be lower than $\bar{s}_{r}$, a negative real parameter chosen by the designer; $T(s, d)$ represents the transfer function from reference $I_{b}^{*}$ to current $I_{b}$, aka the complementary sensitivity function of the converter ${ }^{2}$.

The robustness specification focuses on unstructured uncertainty, e.g. resulting from the modeling approximations discussed in Section II. We assume that these approximations introduce an unstructured multiplicative uncertainty in (14), characterized by $G_{w}(s, d)\left(1+W_{T}(s) \Delta(s)\right)$, where $W_{T}$ represents a frequency-dependent uncertainty profile and $\Delta$ a multiplicative uncertainty satisfying $\|\Delta\|_{\infty} \leq 1$. By direct application of the small-gain Theorem, robustness against this uncertainty can be ensured as long as [16]

$$
\left\|W_{T}(s) T(s, d)\right\|_{\infty} \leq 1
$$

In this work the weight $W_{T}$ is defined as $W_{T}=h_{T}\left(s+\omega_{T} l_{T}\right) /\left(s+\omega_{T} h_{T}\right)$, where $l_{T}$, $h_{T}$ represent the (relative) model uncertainty at low and high frequencies, respectively, and $\omega_{T}$ the cross-over frequency. Table I contains the values of the specifications employed in this work.

$$
{ }^{2} T(s, d)=\frac{G_{w}(s, d) C_{w}(s, d)}{1+G_{w}(s, d) C_{w}(s, d)} .
$$
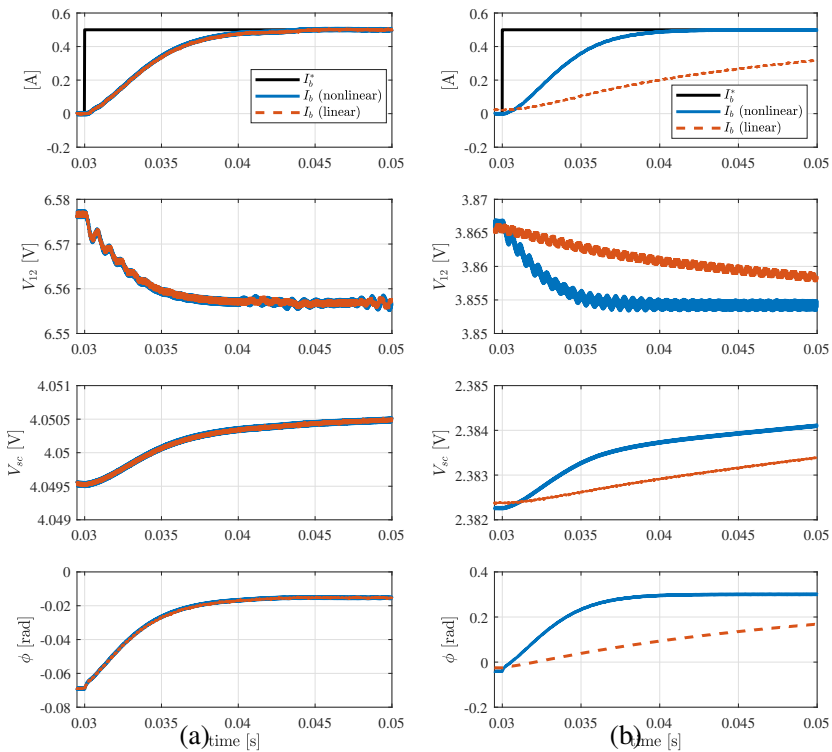

Fig. 4. Numerical validation of the current controller: step response with duty cycle $d=0.5$ (a) and $d=0.8(\mathrm{~b})$.

4) Synthesis: The controller synthesis is the last step in the design of the current controller. The aim is to find the set of control parameters $\left(k_{c}, \zeta_{z}, \omega_{z}\right)$ that fulfill the specifications described in the previous sub-section. To facilitate this task, we fix the damping of the controller's zeros $\left(\zeta_{z}=0.707\right)$ and apply the parameter space method to find $\left(k_{c}, \omega_{z}\right)$. The parameter space method maps the limit conditions associated with the control specification into the $\left(k_{c}, \omega_{z}\right)$-plane by exploiting appropriate symbolic and numeric calculations (see [16] for details). It was found that $k_{c}=0.5 \times 10^{-4}, \omega_{z}=2560 \mathrm{rad} / \mathrm{s}$ fulfills the specifications.

\section{Simulation Results}

The proposed nonlinear controller was validated via numerical simulations with the switching model of the DHB presented in Section II. For benchmarking purposes, a baseline current controller based on the linearization of (7) around the equilibrium point $\left(d^{e q}=0.5, \phi^{e q}=0, V_{s c}^{e q}=4 V\right)$ was implemented. This linear controller has the same structure as the $w$-controller and was parameterized with similar closedloop specifications. Appendix A contains the parameters of the model and controller employed in this work.

Fig. 4a shows the result for a step change in the current setpoint with a duty cycle $d=0.5$. One can observe that both linear and nonlinear controllers provide similar responses. This result is justified because: i) both controllers have similar specifications and ii) the linear controller operates close to the equilibrium point employed in the linearization (thus with small modeling errors).

Fig. $4 \mathrm{~b}$ presents the results when the current controllers operate with a large duty cycle $(d=0.8)$. The results reveal that the transient performance of the linear controller degrades significantly in comparison with the nonlinear approach. In 

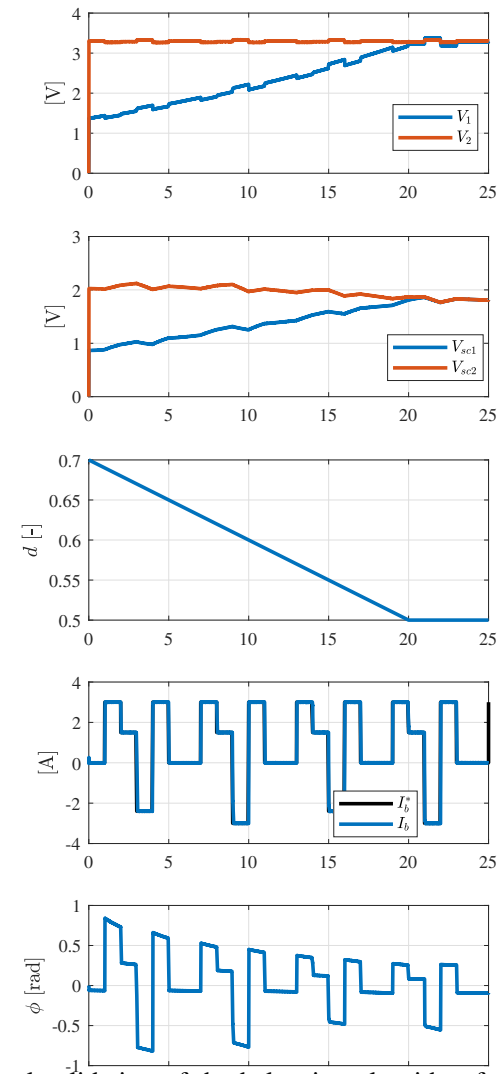

Fig. 5. Numerical validation of the balancing algorithm for the SC voltage.

this case, the linear controller operates further away from the equilibrium point employed in the linearization. Hence, larger modeling errors are introduced, which significantly degrade the transient performance of the linear controller. In contrast, the response of the nonlinear controller remains largely unaltered by the new operating point of the converter.

The second test case focuses on the SC balancing, while following repetitive step-wise $I_{b}^{*}$ profile. This test considers a significant initial imbalance in the SC voltages $\left(V_{s c 1}(0)=\right.$ $\left.0.85 \mathrm{~V}, V_{s c 2}(0)=2 \mathrm{~V}\right)$, which might appear due to differences in the self-discharge rates of the SCs. As depicted in Fig. 5, the SC balancing algorithm initializes the converter's duty cycle to match the initial voltage in the SCs $(d=0.7)$. Afterwards, the balancing algorithm slowly decreases the duty cycle $d$ in order to equalize the SC voltage, which is successfully achieved after 20 s.

\section{CONClusion}

We proposed a nonlinear framework for controlling dual half bridge power converters. This framework employed feedback linearization methods to track current in the converter's primary side, while a pragmatic balancing algorithm manipulated the duty cycle as a means to eliminate voltage mismatches in the converter's secondary ports. The ability to cancel the dominant nonlinearities in the converter represents one of the key advantages offered by the proposed approach, which facilitates the control design task. Future work will focus on the experimental validation of the nonlinear control framework and its potential application in hybrid battery balancing systems.

\section{ACKNOWLEDGMENT}

The work of R. de Castro and J. Brembeck was funded by the project DLR-Next Generation Car, Antriebsstrang und Energiemanagement.

\section{APPENDIX}

\section{A. Converter and ESS Parameters}

ESS: $v_{b}=3.3 \mathrm{~V}, C_{s c 1}=C_{s c 2}=0.350 \mathrm{~F}$, DHB: $R_{b}=$ $10 \mathrm{~m} \Omega, L_{b}=33 \mu \mathrm{H}, C_{1}=C_{2}=0.220 m F, L_{r}=1.7 \mu \mathrm{H}$, $\omega=2 \pi 20 \mathrm{kHz}$,

\section{REFERENCES}

[1] E. Chemali, M. Preindl, P. Malysz, and A. Emadi, "Electrochemical and electrostatic energy storage and management systems for electric drive vehicles: State-of-the-Art review and future trends," IEEE Journal of Emerging and Selected Topics in Power Electronics, vol. 4, no. 3, pp. 1117-1134, Sep. 2016.

[2] T. Baumhöfer, M. Brühl, S. Rothgang, and D. U. Sauer, "Production caused variation in capacity aging trend and correlation to initial cell performance," J. Power Sources, vol. 247, pp. 332-338, Feb. 2014.

[3] Hui Li and F. Z. Peng, "Modeling of a new ZVS bi-directional dc-dc converter," IEEE Trans. Aerosp. Electron. Syst., vol. 40, no. 1, pp. 272 283, Jan. 2004.

[4] F. Gao, N. Mugwisi, and D. Rogers, "Three degrees of freedom operation of a dual half bridge," in 21st European Conference on Power Electronics and Applications, 2019.

[5] R. de Castro, C. Pinto, J. Varela Barreras, R. E. Araújo, and D. A. Howey, "Smart and hybrid balancing system: Design, modeling, and experimental demonstration," IEEE Trans. Veh. Technol., vol. 68, no. 12, pp. 11449-11 461, Dec. 2019.

[6] P. He and A. Khaligh, "Comprehensive analyses and comparison of 1 kw isolated DC-DC converters for bidirectional EV charging systems,' IEEE Transactions on Transportation Electrification, vol. 3, no. 1, pp. 147-156, Mar. 2017.

[7] F. Gao and D. Rogers, "Duty-cycle plus phase-shift control for a dual active half bridge based bipolar DC microgrid," in 2018 IEEE Applied Power Electronics Conference and Exposition, 2018.

[8] Hui Li, Fang Zheng Peng, and J. S. Lawler, "A natural ZVS mediumpower bidirectional DC-DC converter with minimum number of devices," IEEE Trans. Ind. Appl., vol. 39, no. 2, pp. 525-535, Mar. 2003.

[9] M. S. Irfan, A. Ahmed, J. Park, and C. Seo, "Current-Sensorless PowerDecoupling Phase-Shift Dual-Half-Bridge converter for DC-AC power conversion systems without electrolytic capacitor," IEEE Trans. Power Electron., vol. 32, no. 5, pp. 3610-3622, May 2017.

[10] K. Xiangli, S. Li, and K. M. Smedley, "Decoupled PWM plus PhaseShift control for a Dual-Half-Bridge bidirectional DC-DC converter," IEEE Trans. Power Electron., vol. 33, no. 8, pp. 7203-7213, Aug. 2018.

[11] S. Chakraborty and S. Chattopadhyay, "Minimum-RMS-Current operation of asymmetric dual active Half-Bridge converters with and without ZVS," IEEE Trans. Power Electron., vol. 32, no. 7, pp. 5132-5145, Jul. 2017.

[12] F. M. Ibanez, "Analyzing the need for a balancing system in supercapacitor energy storage systems," IEEE Trans. Power Electron., vol. 33, no. 3, pp. 2162-2171, Mar. 2018.

[13] F. de Leon, A. Farazmand, and P. Joseph, "Comparing the T and $\pi$ equivalent circuits for the calculation of transformer inrush currents," IEEE Trans. Power Delivery, vol. 27, no. 4, pp. 2390-2398, Oct. 2012.

[14] J. Sun and H. Grotstollen, "Averaged modelling of switching power converters: reformulation and theoretical basis," in 23rd Annual IEEE Power Electronics Specialists Conference, 1992.

[15] H. K. Khalil, Nonlinear Systems, ser. 3rd ed. Upper Saddle River, NJ: Prentice-Hall, 2002.

[16] J. Ackermann, Robust Control: The Parameter Space Approach. Springer, 2002. 